\title{
MEDIAL PATELLOFEMORAL LIGAMENT RECONSTRUCTION TO TREAT RECURRENT PATELLAR DISLOCATION
}

Matheus Braga Jacques Gonçalves', Lúcio Honório de Carvalho Júnior², Luiz Fernando Machado Soares', Tiago Jacques Gonçalves' ${ }^{1}$ Rogério Luciano dos Santos ${ }^{3}$, Marcelo Lobo Pereira ${ }^{4}$

\section{ABSTRACT}

Objective: To present a new technique for reconstruction of the medial patellofemoral ligament (MPFL) in patients with recurrent patellar dislocation and to evaluate the clinical findings from this. Methods: Between January 2007 and January 2008, 23 patients underwent reconstruction of the MPFL with a free graft from the semitendinosus tendon. After a minimum of 24 months of follow-up, 22 patients were evaluated using the Kujala and Lysholm clinical protocols. Results: The mean follow up was 26.2 months. According to the Lysholm protocol, the patients had a mean score of 53.72 points preoperatively and 93.36 points postoperatively $(\mathrm{p}=0.000006)$. According to the Kujala protocol, the mean score was 59.81 points preoperatively and 83.54 points postoperatively ( $p=$ 0.002173). Conclusion: Reconstruction of the medial patellofemoral ligament using the proposed technique showed excellent results over the short term, when evaluated by means of clinical protocols.

Keywords - Joint Instability; Knee Injuries; Patellar Ligament; Patellofemoral Joint

\section{INTRODUCTION}

Dislocations of the patella are common, and tend to occur as a result of contracting the quadriceps during weight-bearing, with valgus flexion of the knee and the tibia rotated externally.

According to McManus, the natural history of dislocating patella that is not treated, or is treated conservatively, involves, redislocation in one out six cases, and residual symptoms in $33 \%$, with only half of patients becoming asymptomatic. In $25 \%$ of cases, there is a family history of dislocating patella ${ }^{(1)}$.

The role of the medial patellofemoral ligament (MPFL) as the primary restrictor of dislocating patella has been described by various authors ${ }^{(2-6)}$. Studies on cadavers have proven that this ligament acts by blocking the lateral forces ${ }^{(2,5,7)}$. Rupture of this structure was found in eight out of 10 cases of experimentally produced dislocations in cadavers ${ }^{(2)}$ and in 15 out of 16 cases studied in vivo ${ }^{(8)}$. Davis and Fithian demonstrated that insufficiency of this ligament is a determining factor of patellar instability, if any other predisposing factor is present ${ }^{(9)}$.

The objective of this study is to present a new technique for reconstruction of the medial patellofemoral ligament, using a free semitendinosus tendon graft, and to evaluate the results of patients operated on through two clinical protocols.

1 - Orthopedic Doctor, Member of the Knee Group of the Hospital Madre Teresa, MG, Brazil.

2 - Doctorate from Unifesp; Assistant Professor of the Locomotor Apparatus Department of the UFMG School of Medicine. Member of the Knee Group of the Hospital Madre Teresa, MG, Brazil.

3 - Student Intern, Member of the Knee Group of the Hospital Madre Teresa, MG, Brazil.

4 - Specializing Doctor at the Hospital Madre Teresa, MG, Brazil.

Work carried out at the Hospital Madre Teresa, MG, Brazil.

Correspondence: R. Olavo Carsalade Vilela, 264, Ipê da Serra - 34000-000 - Nova Lima, MG. E-mail: luciohcj@medicina.ufmg.br

Work received for publication: March 5, 2010; accepted for publication: May 4, 2010. 


\section{METHODS}

In the period from January 2007 to January 2008, 23 patients with recurrent dislocating patella were operated on by the authors, at the Hospital Madre Teresa (HMT) in Belo Horizonte. The average age of the patients was 28.6 years, with ages ranging from 16 to 45 years. Eight patients were male and 14 female. All the patients underwent radiological and tomographic evaluations before surgery. In all of the patients, isolated medial patellofemoral ligament reconstruction was performed using free semitendinosus tendon graft, operated according to the technique presented by the authors.

All the patients presented dysplasia of the femoral trochlea, as described by Dejour et al ${ }^{(10)}$. None of them presented a distance between the anterior tibial tuberosity and the trochlear throat (AT-TT) of more than $20 \mathrm{~mm}$, or high-riding patella, both of which were exclusion criteria.

Twenty-two operated patients were available for evaluation. All of them were evaluated pre-and postoperative, using the clinical protocols of Kujala et $\mathrm{al}^{(11)}$ and Tegner and Lysholm ${ }^{(12)}$.

The difference between the means was statistically analyzed using measures of central tendency by the Student-t test, through the program Epi Info version 6.04, considering a statistical significance of $p$ less than 0.05 .

The research project was approved by the Research Ethics Committee of the HMT.

\section{SURGICAL TECHNIQUE}

The procedures were carried out under epidural block or rachianesthesia, without concomitant block of the femoral nerve. The patients were positioned in the supine position, with a pneumatic tourniquet in the root of the thigh. After removing the tendon of the semitendinosus muscle through an access incision of 2 to $3 \mathrm{~cm}$ medially to the anterior tibial tuberosity, an incision of the same size was made in the medial side of the patella, in the proximal third (Figures 1a and 1b). Through this incision, two bone tunnels were created in the patella, $2 \mathrm{~cm}$ distal to the insertion of the quadriceps tendon. The first tunnel was made on the anterior side of the patella $1 \mathrm{~cm}$ from its medial border. The second tunnel was made on the medial side of the patella, joining the anterior tunnel at a 90 degree angle (Figures $1 \mathrm{c}$ and 2a). The diameters of these tunnels ranged from 3.5 to $4.5 \mathrm{~mm}$, depending on the diameter of the semitendinosus tendon.

After preparation of the graft obtained, one of its ends was introduced into the patellar tunnels and the other in the tunnel created, with mixter forceps, in the medial and distal third of the quadriceps tendon, close to its insertion point (Figure 1d). The two ends were then sutured together and passed under the vastus medialis muscle, between the second and third layers of the retinaculum, and then to the medial epicondyle region, where a third access of 2 to $3 \mathrm{~cm}$ had been prepared (Figures 1e and 1f).

At this point, slightly proximal and posterior to the medial epicondyle (Nomura's point) ${ }^{(13)}$, a guide
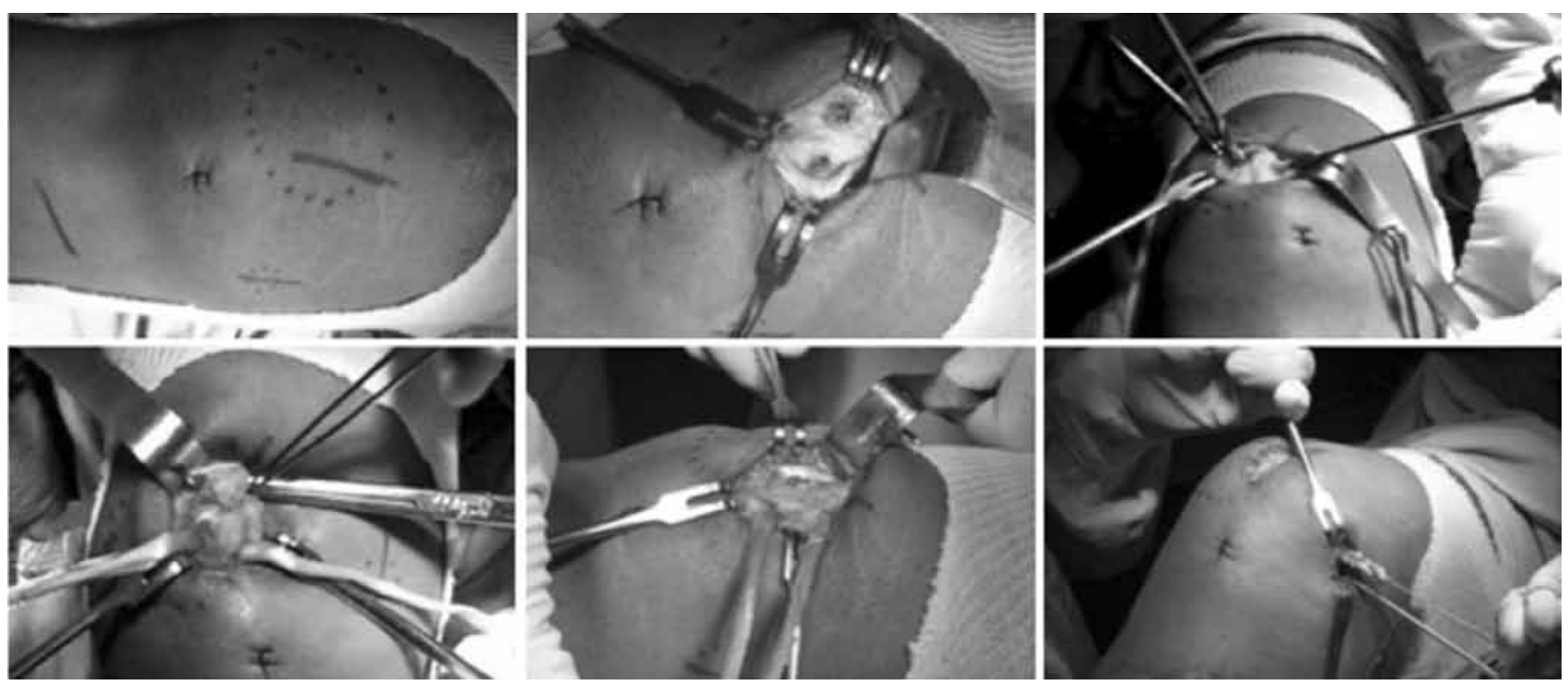

Figure 1 - Technique: preparation of the tunnels through which the graft is passed. 
wire was introduced. After testing the isometry of the graft, a femoral bone tunnel was then prepared, with the same diameter as the sutured ends of the tendon. These were there introduced and tensioned with the knee at 60 degrees of flexion. The graft was fixed at this site, with an interference screw of the same diameter (Figures $2 b$ and $2 c$ ).

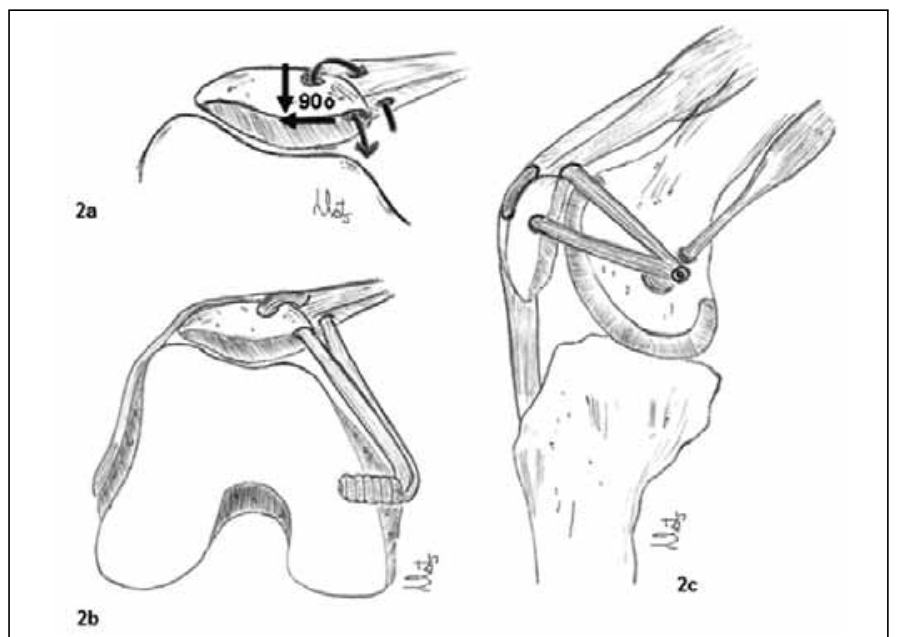

Figure 2 - Reconstruction of the MPFL with patellar tunnel and transquadriceps tunnel.

After the femoral fixation, the stability obtained and the range of motion were tested. There was no lateral retinacular release in any of the patients. After closing, the knee was then immobilized in extension with a removable immobilizer, which was progressively removed over a period of six weeks.

\section{RESULTS}

The mean duration of symptoms to surgery was 141 months, ranging from two to 360 months. The mean follow-up of the patients was 26.1 months, ranging from 24 to 32 months.

According to the Lysholm protocol, the average score of the patients prior to surgery was 53.72 points, ranging from 29 to 77 . After surgery, the average score for the same patients was 93.36, ranging from 69 to 100 points. The difference between the average scores was statistically significant, with a value of $p=0.000006$.

According to the Kujala protocol, the average score of the patients prior to surgery was 59.81 points, ranging from 32 to 88 . After surgery, the average score for the same patients was 83.54, ranging from 71 to 96 points. The difference between the averages was statistically significant, with a value of $p=0.002173$.

Rev Bras Ortop. 2011;46(2):160-4

\section{DISCUSSION}

The medial stabilizers of the patella include the superficial medial retinaculum, medial patellofemoral ligament (MPFL), medial patellotibial ligament, medial patellomeniscal ligament, and the oblique vastus medialis ${ }^{(13,14)}$. Biomechanical studies have indicated the MPFL as the primary restrictor of lateralization of the patella, contributing to $50 \%$ to $80 \%$ of the medial contention, according to different authors ${ }^{(5,7,13,14-16)}$.

Amis et $\mathrm{al}^{(17)}$ demonstrated that the MPFL has a mean tensile strength of $208 \mathrm{~N}$. However, its limited lengthening capacity results in its total rupture in cases of complete dislocating patella, as shown in a biomechanical study by Mountney et $\mathrm{al}^{(18)}$. This fact was confirmed by other studies, in which rupture of the ligament was found in eight out of 10 cases of experimentally produced dislocations in cadavers ${ }^{(2)}$ and in 15 of 16 cases studied in vivo ${ }^{(8)}$. Magnetic resonance exams have also confirmed lesion of the MPFL in the majority of cases of acute dislocating patella ${ }^{(19)}$. Ligament insufficiency is present in all cases of recurrent dislocation, according to the same authors.

More than a hundred procedures for the treatment of recurrent dislocation of the patella have been developed over the last century ${ }^{(13)}$. The majority of these techniques seek to realign the extensor mechanism, reducing lateralization of the patella when the quadriceps is activated ${ }^{(3,4,6,7)}$. However, distal realignment procedures, with medialization of the anterior tibial tuberosity, have shown limited clinical success ${ }^{(6,20)}$. Proximal realignment procedures depend on the contraction of the quadriceps to maintain the patella in the trochlear sulcus. By contrast, intact passive stabilizers, such as the MPFL, appear to have a predominant role, independent of misalignment ${ }^{(6)}$.

In view of this evidence of the superiority of the use of the MPFL, various authors have defended its reconstruction for the treatment of patellar instability, as it appears to be more effective than proximal or distal realignment techniques ${ }^{(6,8,16,21,22)}$.

The knowledge of the anatomy of the MPFL is crucial for its reconstruction. Nomura et $\mathrm{al}^{(13)}$ are the authors who best describe its anatomy, and according to them, some fibers of the MPFL extend upwards, beyond the upper edge of the patella, directly into the quadriceps tendon. The ligament also receives the insertion of the oblique vastus medialis muscle at its patellar end, in a 
portion extending for around $35 \%$ of its total length ${ }^{(13,17)}$ It is believed that when the muscle contracts, the MPFL can be pulled in a proximal direction, becoming more tense. This suggests that the oblique vastus medialis and the MPFL probably act together as a combined dynamic complex ${ }^{(13,23)}$.

MPFL reconstruction techniques using free autologous graft of the flexor tendons enable its positioning in the anatomical points of the ligament. Schöttle et $\mathrm{al}^{(15)}$ believe that these points cannot be reached with techniques with "tilted" tendons, but Camanho et $\mathrm{al}^{(24)}$, through the technique with patellar tendon, have demonstrated otherwise.

The technique proposed here uses a narrow tunnel in a small area of the patella, enabling its passage at the medial and anterior borders of the patella to be controlled more easily, and minimizing the potential complications. The second tunnel is created through the quadriceps tendon, at the upper margin of the patella, thereby maintaining the anatomical insertion of the MPFL ${ }^{(13)}$ and taking advantage of the dynamic combined action of the oblique medialis vastus muscle.

There is much discussion on the isometry of the native MPFL, and how the graft should behave during the arc of movement of the knee $\mathrm{e}^{(16,23-28)}$. The normal ligament presents greater tension when fully extended with the quadriceps contracted, but the ideal variation in length for the MPFL graft has still not been established $^{(29)}$.

The angle of flexion of the knee during the fixation and tensioning of the graft is also a point of controversy.
Fixations at $0^{\circ}, 30^{\circ}, 45^{\circ}$ and $90^{\circ}$ have been recommend$\mathrm{ed}^{(15,21,31,39)}$. Nomura et $\mathrm{al}^{(16,32)}$ evaluated the alteration in length of the MPFL throughout the arc of movement, and showed that its length is close to the maximum in extension, and at $60^{\circ}$ of flexion. In extension, the patella is not inserted in the trochlear sulcus, and determining its correct position during surgery is difficult. However, at $60^{\circ}$ of knee flexion, the trochlear sulcus is sufficiently deep and the appropriate position of the patella is easy to determine manually.

MPFL reconstruction has produced good patellar stability, regardless of the technique used. Lind et $\mathrm{al}^{(33)}$, in a review article, observed an absence of new dislocations in five out of eight studies, and redislocation rates of less than $7 \%$ in the remaining three studies, which can be considered a successful result compared with other surgical patellar stabilization techniques, in which redislocation rates of $10 \%$ to $35 \%$ have been described ${ }^{(9)}$.

This study presents the case studies and results comparable to other works in the literature, using the semitendinosus tendon graft ${ }^{(14,30,31,34-36)}$. With zero levels of redislocation and success rates based on clinical protocols achieving scores of 80-90 out of 100 possible points, as shown in Table 1.

\section{CONCLUSION}

Reconstruction of the medial patellofemoral ligament with free semitendinosus tendon graft has shown, in the short-term, excellent results when evaluated by clinical protocols.

Table 1 - Results of clinical trials evaluating MPFL reconstruction.

\begin{tabular}{|c|c|c|c|c|c|c|}
\hline Author & Graft & Fixation & $\begin{array}{l}\text { No. of } \\
\text { patients }\end{array}$ & Follow-up & Redislocation & Clinical score \\
\hline Ellera Gomes 1992 & Artificial ligament & $\begin{array}{l}\text { Patellar bone tunnel, } \\
\text { metallic femoral screw }\end{array}$ & 30 & 39 months & Zero & $\begin{array}{l}\text { Crosby Insall } 84 \% \text { Good } \\
\text { and excellent }\end{array}$ \\
\hline $\begin{array}{l}\text { Nomura and Inoue } \\
2003\end{array}$ & Artificial ligament & $\begin{array}{l}\text { Patellar bone tunnel, } \\
\text { femoral staple }\end{array}$ & 27 & $5-9$ years & $4 \%$ & $\begin{array}{l}\text { Crosby Insall } 96 \% \text { Good } \\
\text { and excellent }\end{array}$ \\
\hline Deie et al 2005 & Semitendinosus & Suture, patella and femur & 39 & 5 years & Zero & Kujala 92 \\
\hline Steiner et al 2006 & $\begin{array}{l}\text { Autologous graft } \\
\text { of the adductor }\end{array}$ & $\begin{array}{l}\text { Patellar bone tunnel, } \\
\text { femoral suture }\end{array}$ & 34 & 2-10 years & Zero & Kujala 90 \\
\hline Mikashima et al 2006 & Semitendinosus & $\begin{array}{l}\text { Suture and patellar bone } \\
\text { tunnel, femoral screw }\end{array}$ & 24 & 2 years & Zero & $\begin{array}{l}\text { No difference between } \\
\text { suture and patellar tunnel }\end{array}$ \\
\hline Watanabe et al 2008 & Semitendinosus & $\begin{array}{l}\text { Patellar suture, femoral } \\
\text { endobutton }\end{array}$ & 42 & 4.3 years & Not mentioned & Lysholm 92 \\
\hline $\begin{array}{l}\text { Christiansen et al } \\
2008\end{array}$ & Gracilis & $\begin{array}{l}\text { Patellar bone tunnel, } \\
\text { femoral screw }\end{array}$ & 45 & 2 years & $2 \%$ & Kujala 86 \\
\hline \multirow{2}{*}{ Gonçalves et al 2010} & \multirow{2}{*}{ Semitendinosus } & \multirow{2}{*}{$\begin{array}{l}\text { Patellar bone tunnel, } \\
\text { femoral screw }\end{array}$} & \multirow{2}{*}{22} & \multirow{2}{*}{26 months } & \multirow{2}{*}{ Zero } & Kujala 83.5 \\
\hline & & & & & & Lysholm 93.3 \\
\hline
\end{tabular}




\section{REFERENCES}

1. McManus F, Rang M, Heslin DJ. Acute dislocation of the patella in children. The natural history. Clin Orthop Relat Res. 1979;(139):88-91.

2. Burks RT, Desio SM, Bachus KN, Tyson L, Springer K. Biomechanical evaluation of lateral patellar dislocations. Am J Knee Surg. 1998;11(1):24-31.

3. Conlan T, Garth WP Jr, Lemons JE. Evaluation of the medial soft-tissue restraints of the extensor mechanism of the knee. J Bone Joint Surg Am. 1993;75(5):682-93.

4. Cox JS. Evaluation of the Roux-EImslie-Trillat procedure for knee extensor realignment. Am J Sports Med. 1982;10(5):303- 10.

5. Desio SM, Burks RT, Bachus KN. Soft tissue restraints to lateral patellar translation in the human knee. Am J Sports Med. 1998;26(1):59-65.

6. Schöttle PB, Fucentese SF, Romero J. Clinical and radiological outcome of medial patellofemoral ligament reconstruction with a semitendinosus autograft for patella instability. Knee Surg Sports Traumatol Arthrosc. 2005;13(7):516-21.

7. Hautamaa PV, Fithian DC, Kaufman KR, Daniel DM, Pohlmeyer AM. Medial soft tissue restraints in lateral patellar instability and repair. Clin Orthop Relat Res. 1998;(349):174-82.

8. Sallay PI, Poggi J, Speer KP, Garrett WE. Acute dislocation of the patella. A correlative pathoanatomic study. Am J Sports Med. 1996;24(1):52-60.

9. Davis DK, Fithian DC. Techniques of medial retinacular repair and reconstruction. Clin Orthop Relat Res. 2002;(402):38-52

10. Dejour H, Walch G, Neyret P, Adeleine P. [Dysplasia of the femoral trochlea] Rev Chir Orthop Reparatrice Appar Mot. 1990;76(1):45-54.

11. Kujala UM, Jaakkola LH, Koskinen SK, Taimela S, Hurme M, Nelimarkka O. Scoring of patellofemoral disorders. Arthroscopy. 1993;9(2):159-63.

12. Tegner $Y$, Lysholm J. Rating systems in the evaluation of knee ligament injuries. Clin Orthop Relat Res. 1985;(198):43-9..

13. Nomura E, Inoue M, Osada N. Anatomical analysis of the medial patellofemoral ligament of the knee, especially the femoral attachment. Knee Surg Sports Traumatol Arthrosc. 2005;13(7):510-5.

14. Christiansen SE, Jacobsen BW, Lund B, Lind M. Reconstruction of the medial patellofemoral ligament with gracilis tendon autograft in transverse patellar drill holes. Arthroscopy. 2008;24(1):82-7.

15. Schöttle P, Schmeling A, Romero J, Weiler A. Anatomical reconstruction of the medial patellofemoral ligament using a free gracilis autograft. Arch Orthop Trauma Surg. 2009;129(3):305-9.

16. Nomura E, Horiuchi Y, Kihara M. Medial patellofemoral ligament restraint in lateral patellar translation and reconstruction. Knee. 2000;7(2):121-127.

17. Amis AA, Firer P, Mountney J, Senavongse W, Thomas NP. Anatomy and biomechanics of the medial patellofemoral ligament. Knee. 2003;10(3):215-20.

18. Mountney J, Senavongse W, Amis AA, Thomas NP. Tensile strength of the medial patellofemoral ligament before and after repair or reconstruction. J Bone Joint Surg Br. 2005;87(1):36-40.

19. Nomura E. Classification of lesions of the medial patello-femoral ligament in patellar dislocation. Int Orthop. 1999;23(5):260-3.
20. Aglietti P, Buzzi R, De Biase P, Giron F. Surgical treatment of recurrent dislocation of the patella. Clin Orthop Relat Res. 1994;(308):8-17.

21. Drez D Jr, Edwards TB, Williams CS. Results of medial patellofemoral ligament reconstruction in the treatment of patellar dislocation. Arthroscopy. 2001;17(3):298-306.

22. Muneta T, Sekiya I, Tsuchiya M, Shinomiya K. A technique for reconstruction of the medial patellofemoral ligament. Clin Orthop Relat Res. 1999;(359):151-5.

23. Mulford JS, Wakeley CJ, Eldridge JD. Assessment and management of chronic patellofemoral instability. J Bone Joint Surg Br. 2007;89(6):709-16.

24. Camanho GL, Bitar AC, Hernandez AJ, Olivi R. Medial patellofemoral ligament reconstruction: a novel technique using the patellar ligament. Arthroscopy. 2007;23(1):108.e1-4.

25. Ellera Gomes JL, Stigler Marczyk LR, César de César P, Jungblut CF. Medial patellofemoral ligament reconstruction with semitendinosus autograft for chronic patellar instability: a follow-up study. Arthroscopy. 2004;20(2):147-51.

26. Schock EJ, Burks RT. Medial patellofemoral ligament reconstruction using a hamstring graft. Oper Tech Sports Med. 2001;9(3):169-175.

27. Smirk $\mathrm{C}$, Morris $\mathrm{H}$. The anatomy and reconstruction of the medial patellofemoral ligament. Knee. 2003;10(3):221-7.

28. Steensen RN, Dopirak RM, McDonald WG 3rd. The anatomy and isometry of the medial patellofemoral ligament: implications for reconstruction. Am J Sports Med. 2004;32(6):1509-13.

29. Fithian DC, Gupta N. Patella instability: principals of soft tissue repair and reconstruction. Tech Knee Surg. 2006;5(1):19-26.

30. Deie M, Ochi M, Sumen Y, Adachi N, Kobayashi K, Yasumoto M. A long-term follow-up study after medial patellofemoral ligament reconstruction using the transferred semitendinosus tendon for patellar dislocation. Knee Surg Sports Traumatol Arthrosc. 2005;13(7):522-8.

31. Ellera Gomes JL. Medial patellofemoral ligament reconstruction for recurrent dislocation of the patella: a preliminary report. Arthroscopy. 1992;8(3):335-40.

32. Nomura E, Inoue M. Hybrid medial patellofemoral ligament reconstruction using the semitendinous tendon for recurrent patellar dislocation: minimum 3 years' follow-up. Arthroscopy. 2006;22(7):787-93.

33. Lind M, Jakobsen BW, Lund B, Christiansen SE. Reconstruction of the medial patellofemoral ligament for treatment of patellar instability. Acta Orthop. 2008;79(3):354-60.

34. Steiner TM, Torga-Spak R, Teitge RA. Medial patellofemoral ligament reconstruction in patients with lateral patellar instability and trochlear dysplasia. Am J Sports Med. 2006;34(8):1254-61.

35. Mikashima Y, Kimura M, Kobayashi Y, Miyawaki M, Tomatsu T. Clinical results of isolated reconstruction of the medial patellofemoral ligament for recurrent dislocation and subluxation of the patella. Acta Orthop Belg. 2006;72(1):65-71.

36. Watanabe T, Muneta T, Ikeda H, Tateishi T, Sekiya I. Visual analog scale assessment after medial patellofemoral ligament reconstruction: with or without tibial tubercle transfer. J Orthop Sci. 2008;13(1):32-8. 